Southern Illinois University Carbondale

OpenSIUC

Publications

Department of Physics

7-15-2004

Size Induced Variations in Structural and Magnetic Properties of Double Exchange La0.8Sr0.2MnO3- $\delta$ Nano-Ferromagnet

Sujoy Roy

University of California - San Diego

Igor Dubenko

Southern Illinois University Carbondale

Dossah D. Edorh

Southern Illinois University Carbondale

Naushad Ali

Southern Illinois University Carbondale

Follow this and additional works at: http://opensiuc.lib.siu.edu/phys_pubs

(C) 2004 American Institute of Physics

Published in Journal of Applied Physics, Vol. 96 No. 2 (2004) at doi: 10.1063/1.1760230

Recommended Citation

Roy, Sujoy, Dubenko, Igor, Edorh, Dossah D. and Ali, Naushad. "Size Induced Variations in Structural and Magnetic Properties of Double Exchange La0.8Sr0.2MnO3- $\delta$ Nano-Ferromagnet." (Jul 2004).

This Article is brought to you for free and open access by the Department of Physics at OpenSIUC. It has been accepted for inclusion in Publications by an authorized administrator of OpenSIUC. For more information, please contact opensiuc@lib.siu.edu. 


\title{
Size induced variations in structural and magnetic properties of double exchange $\mathrm{La}_{0.8} \mathrm{Sr}_{0.2} \mathrm{MnO}_{3-\delta}$ nano-ferromagnet
}

\author{
Sujoy Roy, ${ }^{\text {a) }}$ Igor Dubenko, Dossah D. Edorh, and Naushad Alib) \\ Department of Physics, Southern Illinois University, Carbondale, Illinois 62901
}

(Received 9 June 2003; accepted 21 April 2004)

\begin{abstract}
A detailed study on the influence of particle size varied from $8 \mathrm{~nm}$ to $53 \mathrm{~nm}$ on the structural and magnetic properties of $\mathrm{La}_{0.8} \mathrm{Sr}_{0.2} \mathrm{MnO}_{3-\delta}$ has been done. The unit cell volume increases and the microstrain in the compound shows peak formation as the particle size decreases. Nano particles of $\mathrm{La}_{0.8} \mathrm{Sr}_{0.2} \mathrm{MnO}_{3-\delta}$ exhibit superparamagnetism whose blocking temperature has a nonlinear and logarithmic decreasing tendency as function of particle size and applied magnetic field, respectively. Evidence of formation of a magnetically dead layer at the surface has been found and the ratio of the thickness of the dead layer to the particle size increases exponentially with particle size. The coercivity of the nanoparicles increases manifold as particle size varies from $53 \mathrm{~nm}$ to $21 \mathrm{~nm}$. In the single domain region the coercivity exhibits a $d^{-1.125}$ behavior. The temperature dependence of the saturation magnetization shows strong collective excitation due to the spin wave that varies as $T^{\alpha}$ with $\alpha>\alpha_{\text {bulk }}$ of 3/2. Thus the spin wave does not follow the Bloch law in the case of nano particles of $\mathrm{La}_{0.8} \mathrm{Sr}_{0.2} \mathrm{MnO}_{3-\delta}$. (C) 2004 American Institute of Physics. [DOI: 10.1063/1.1760230]
\end{abstract}

\section{INTRODUCTION}

The magnetic properties of ultrafine granular systems is an interesting subject of research for both theorists and experimentalists. Granular magnetic systems exhibit interesting and complex properties and have the potential for technological applications that includes magnetic recording media, catalysts, medical diagnostics, pigments in paints etc. ${ }^{1-3}$ In the traditional sense, granular ferromagnets are a mixture of two immiscible materials, one of which is a nano sized ferromagnetic particles and the other one is a nonmagnetic material of about the same size. Common examples are granular $\mathrm{Cu}-\mathrm{Co}$ alloy, $\mathrm{Co}-\mathrm{SiO}_{2}$ granular films, etc. ${ }^{4,5}$ Parallel to this, there are also reports on the studies of an ensemble of ultra fine magnetic particles. ${ }^{6-8}$ In both the cases considerable differences in the magnetization has been observed between the bulk and nanoparticles and have been attributed to the presence of nonmagnetic surface layer or due to disordered spin orientation in the surface.

The discovery of large magnetoresistance in the doped manganese perovskite compounds of the form $R_{1-x} A_{x} \mathrm{MnO}_{3}, \quad(R=$ rare earth and $A=$ divalent cation) have attracted much interest due to their potential technological applications. ${ }^{9}$ The ground state of these compounds, is ferromagnetic and metallic and close to $T_{\mathrm{C}}$, undergoes a metalinsulator transition. The properties of these materials are explained by double exchange theory of Zener ${ }^{10}$ and electron lattice interaction. ${ }^{11}$ From an application point of view, the most interesting thing is that near metal-insulator transition these compounds show huge magnetoresistance that leads to the possibility of these materials to be used in technological

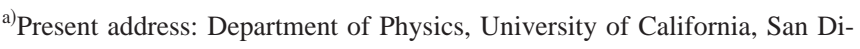
ego, CA.

b) Author to whom correspondence should be addressed; electronic mail: naill@physics.siu.edu
}

applications. However, the requirement of a large applied magnetic field to achieve large magnetoresistance limits practical applicability of the manganese perovskites.

To achieve a low field high magnetoresistance, different extrinsic properties are manipulated. One of them in particular, is the grain size. In granular manganites, the magnetoresistance increases and is related to tunneling between neighboring grains. ${ }^{12,13}$ Very recently, a new approach has been taken where nanopowder of manganite is cold pressed into pellets that allow spin polarized electron transport but not exchange coupling. ${ }^{14}$ Balcells et al. ${ }^{15}$ showed that the low and high field magnetoresistance response of nano grains of $\mathrm{La}_{2 / 3} \mathrm{~S}_{1 / 3} \mathrm{MnO}_{1 / 3}$ could be explained by considering a noncollinear surface layer. Such a model is also suported by LopezQuintela et al. ${ }^{16}$ in their recent work. Magnetic studies performed on ultrafine $\mathrm{La}_{2 / 3} \mathrm{Ca}_{1 / 3} \mathrm{MnO}_{3}$ powder show superparamagnetic behavior and no low field magneoresistance is observed above the superparamagnetic blocking temperature. ${ }^{17}$ Although studies of magnetoresistance have been done, a clear understanding of the magnetic properties of manganite nanoparticles is still lacking, which is essential for proper application purposes.

In the present article we report on the result of our detailed study of the magnetic properties of an ensemble of nanometer scale particles of the canonical double exchange ferromagnet $\mathrm{La}_{0.8} \mathrm{Sr}_{0.2} \mathrm{MnO}_{3-\delta}$ whose grain size varied from 8 to $53 \mathrm{~nm}$. The main objective is to understand the variations of the different structural and magnetic properties as a function of the size of the particle and correlate them. Also information about the special properties of surfaces can be obtained from studies of ultrafine particles as in such a system relatively large fraction of the total number of atom reside in the surface. Our choice of the compound is governed by the fact that $\mathrm{La}_{0.8} \mathrm{Sr}_{0.2} \mathrm{MnO}_{3}$ is a very well studied compound in the bulk form (both single crystal and polycrystal- 
line samples) that allows us to compare the magnetic properties of the nano and the bulk samples. Furthermore, the chemical stability of $\mathrm{La}_{0.8} \mathrm{Sr}_{0.2} \mathrm{MnO}_{3}$ was also given due consideration so that there is minimal chance of disintegration of the compound during the synthesis of nanoparticles from bulk. However, during ball milling it is very likely that the compound loses oxygen. So we have referred our compound as $\mathrm{La}_{0.8} \mathrm{Sr}_{0.2} \mathrm{MnO}_{3-\delta}$.

\section{EXPERIMENTAL PROCEDURE}

Polycrystalline samples of $\mathrm{La}_{0.8} \mathrm{Sr}_{0.2} \mathrm{MnO}_{3-\delta}$ (LSMO) were prepared by the conventional solid state reaction method. Stoichiometric amounts of $\mathrm{La}_{2} \mathrm{O}_{3}, \mathrm{SrCO}_{3}$, and $\mathrm{MnO}_{2}$ powders of $99.9 \%$ or more purity were thoroughly mixed and then calcined for $12 \mathrm{~h}$ at $1000^{\circ} \mathrm{C}$. The resulting powder was then pressed into pellets and sintered at $1280^{\circ} \mathrm{C}$ for $96 \mathrm{~h}$ with intermediate grindings. In order to obtain nanoparticles (NP) of LSMO, the bulk sample was subjected to high energy ball milling using a Fritsch Pulverisette 7 Planetary Mill. The sample was put in tungsten carbide vial and ten $10 \mathrm{~mm}$ tungsten carbide balls were used for grinding. In order to get a narrow and uniform particle size distribution, the grindings were carried out in wet media by pouring few milliliters of ethyl alcohol in the vials. A rotational speed of $500 \mathrm{rpm}$ was chosen and the ball mill was paused every hour to avoid overheating. The sample was ground from $0 \mathrm{~h}$ to 80 $\mathrm{h}$ and a small portion of the sample was taken out after 4,8 , $12,16,48$, and $80 \mathrm{~h}$ for structural and magnetic measurements.

$\mathrm{X}$-ray diffraction (XRD) measurements were performed on all the samples using a Rigaku diffractometer of $12 \mathrm{~kW}$ $\mathrm{x}$-ray generator and $\mathrm{Cu} K \alpha$ radiation. A scanning rate of $0.015^{\circ} \mathrm{s}$ was adopted and the data were taken at room temperature in the $2 \theta$ range of $20-85^{\circ}$. Refinement of the diffraction data was done using the Rietveld powder diffraction profile fitting technique. ${ }^{18}$ The magnetic measurements were done by a Quantum Design SQUID magnetometer in the temperature range of 5-400 $\mathrm{K}$ and applied fields up to $5.5 \mathrm{~T}$. Both zero field cooled (ZFC) and field cooled (FC) magnetization were measured. The transition temperature $T_{\mathrm{C}}$ of the bulk sample was determined from the maxima of the $d M / d T$ versus $T$ curve.

\section{RESULTS AND DISCUSSIONS}

\section{A. Structural properties}

Room temperature $\mathrm{x}$-ray diffraction of bulk LSMO shows the formation of homogeneous single phase compound with rhombohedrally distorted perovskite structure in the space group R3c. Figure 1(a) shows a typical x-ray powder diffraction pattern for the LSMO. The cross points are the actual data and the calculated curve is superposed on it. The short bars are the calculated positions of the reflections for rhombohedral compounds. The bottom line shows the difference between the experimental and the calculated XRD pattern. A goodness of fit factor value of $s=1.46$ indicates a very good agreement between the observed and calculated intensity. Rietveld refinement of the data gives us the lattice parameter values of $a=5.525(3) \AA$ and $c=13.372(3) \AA$.
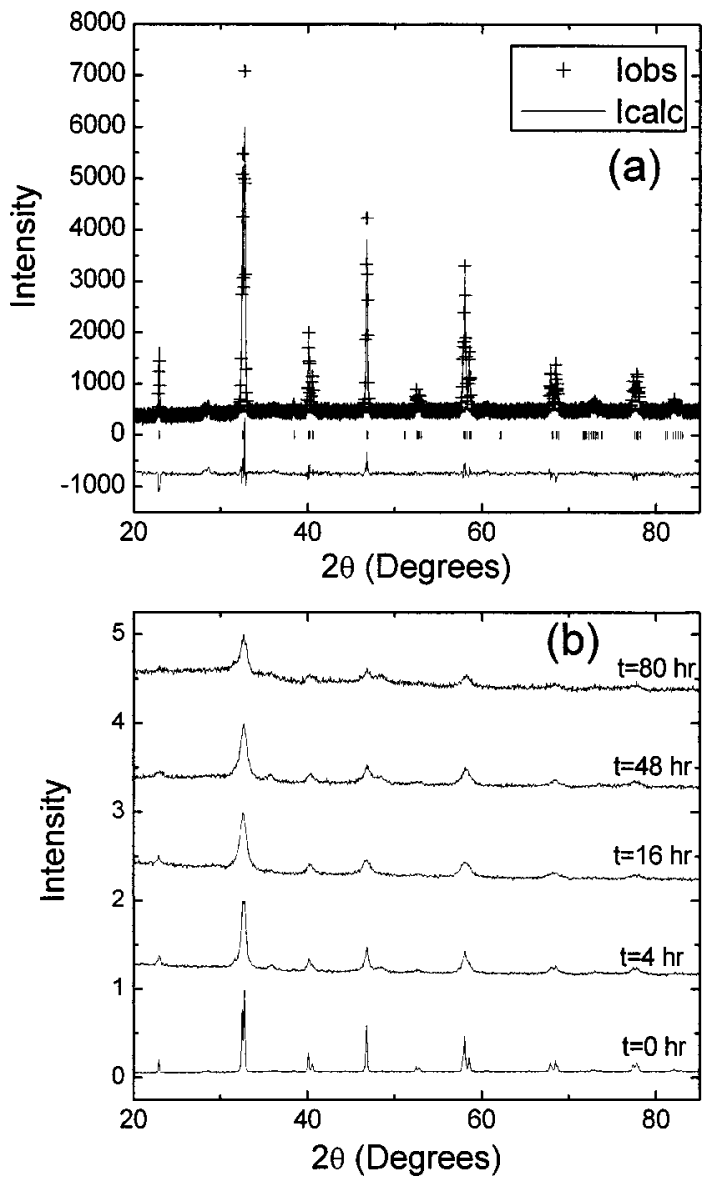

FIG. 1. (a) X-ray diffraction pattern of $\mathrm{La}_{0.8} \mathrm{Sr}_{0.2} \mathrm{MnO}_{3}$. The cross points are the diffractometer data and the continuous line is the refined curve. The bottom line shows the difference curve between the data and the fitted curve. (b) X-ray diffraction pattern of some nanoparticles of $\mathrm{La}_{0.8} \mathrm{Sr}_{0.2} \mathrm{MnO}_{3-\delta}$.

The Mn-O bond length and bond angle were found to be equal to $1.964 \AA$ and $164.3^{\circ}$. These values agree very well with published result [see, for e.g., Ref. 9]. Figure 1(b) shows the XRD pattern of the LSMO compound with increasing milling time (i.e., decreasing grain size). The diffraction pattern gradually broadens out as the particle size goes down. However, the crystalline structure is maintained even for $80 \mathrm{~h}$ milled sample. No additional significant impurity lines are observed to emerge with increasing milling time indicating that the contamination between sample and the vial is negligible. Along with line broadening there is also an increase in background scattering with milling time that is ascribed to an increase in the defects and surface roughness of the NP due to the high energy ball milling process.

Since the diffraction line broadening takes place due to both particle size and $\operatorname{strain}^{19}$ using the simple Scherer equation results in an over estimation of the particle size. Moreover, in the present case, since the high energy ball milling introduces considerable strain in the compound, the contribution of strain to the diffraction line broadening is not negligible. Considering that the particle size and microstrain follow Gaussian distribution, one way to extract the particle size and strain is to use an exact Voigt function as fitting equation. It has been shown that for the above function, by 

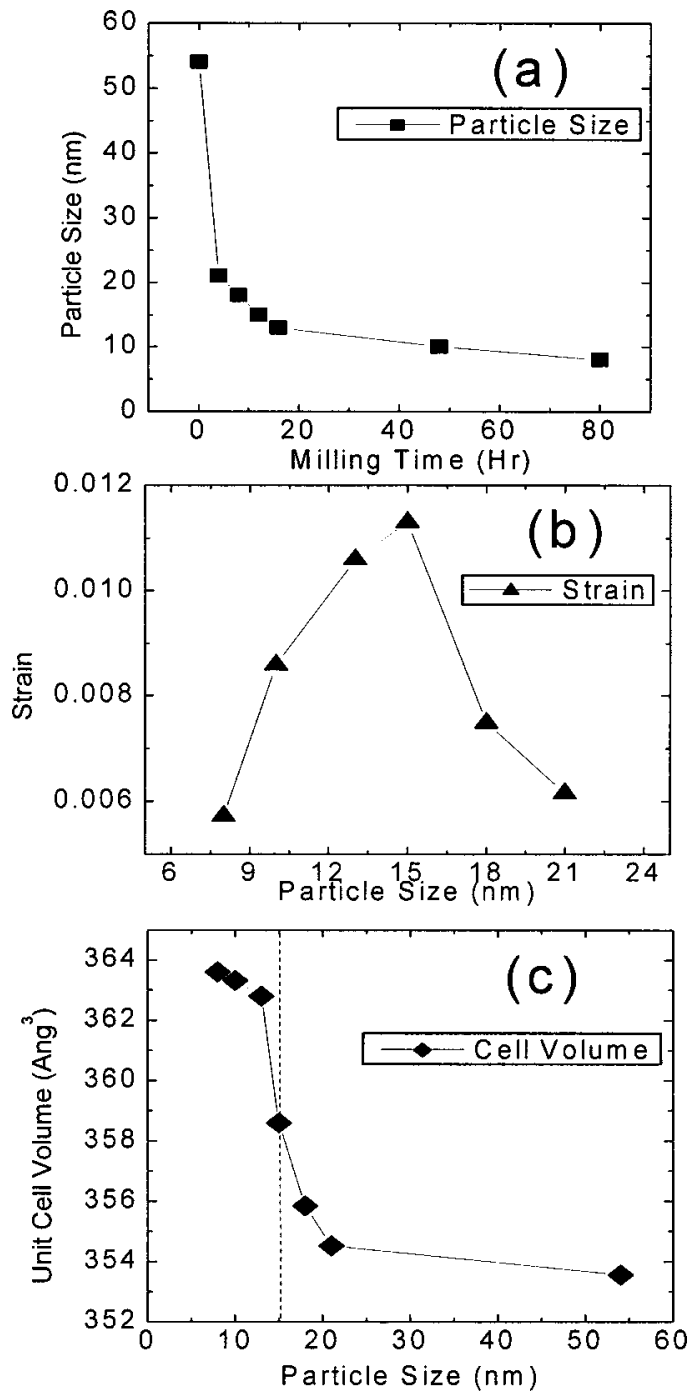

FIG. 2. (a) Variation of the particle size as function of ball milling hours. (b) Evolution of microstrain as function of the particle size. (c) The variation of unit cell volume vs particle size.

using two reflections from the diffraction pattern, the size and strain could be determined using the Warren Averbach relation. Based on the above methodology Balzar ${ }^{20}$ developed a code (BREADTH) to calculate the particle size and strain. The (104) and (024) lines of the diffraction pattern were used to calculate the particle size and microstrain. Figure 2(a) shows the plot of particle size versus milling time. The particle size decreases very rapidly from $53 \mathrm{~nm}(0 \mathrm{~h})$ to $21 \mathrm{~nm}$ during the first $4 \mathrm{~h}$. Then the rate of decrease of size decreases with increasing milling time. As is shown in Fig. 2(a) it takes about $32 \mathrm{~h}$ to decrease the particle size from 10 $\mathrm{nm}$ to $8 \mathrm{~nm}$. Thus it seems that there is a minimum size below which it becomes very difficult to obtain NP of LSMO using the ball mill method. This is quite evident because of the strain hardening that requires increasing energy to break the particles as the size decreases. But since the ball mill can impart only a fixed energy (for constant speed), the minimum size particle is obtained when the energy needed to break the particles become comparable to the strain hardening. This argument also seems to be validated by Hall-Petch relationship ${ }^{21}$ which is given by

$$
\sigma_{\mathrm{y}}=\sigma_{0}+k d^{-1 / 2},
$$

where $\sigma_{\mathrm{y}}$ is the strain, $\sigma_{0}$ and $k$ are constant and $d$ is the average grain diameter. It can be seen from the above equation that the small particle size itself become a hindrance to achieve further refined particles. The roughness of the wall of the vial also plays a role in defining the minimum particle size. For if the particle size becomes smaller than the average roughness of the vial wall, the area of contact between the grinding balls and the grains is very small at the walls. Hence the net impact of the balls on the grains is small whose size then remains unchanged.

Figure 2(b) shows the variation of the microstrain as function of the particle size. The breaking down of the particle results in an increase of the microstrain which attains a maximum value for the particle size of $15 \mathrm{~nm}$ with the microstrain value of $8 \mathrm{~nm}$ and $21 \mathrm{~nm}$ particles being the same. Such a behavior has also been observed for intermetallic compound $\mathrm{AlRu}^{22}$ and $\mathrm{Cu}$ (Ref. 23). In the initial stages of the ball milling, the decrease of the particle size is associated with an increase of the dislocation densities. Since the microstrain generally follows the features of dislocation density, it increases as the grain size decreases. In addition to dislocation, particle size reduction also takes place at a lower stress by a gliding motion along the grain boundaries. ${ }^{24}$ When the particles are big, the grain boundary contribution to the microstrain is very small. However, as the particle size decreases the grain boundary increases relatively and act as barrier to the dislocation motion. As a result the dislocation density reaches saturation and below a particular grain size, further particle size reduction takes place through gliding motion along grain boundaries. The overall effect is then a reduction of the microstrain as shown in Fig. 2(b).

The unit cell volume increases as the particle size reduces [Fig. 2(c)]. The unit cell volume increases slowly in the initial stages of the ball milling process and for the particle sizes below $15 \mathrm{~nm}$ the unit cell volume increases abruptly. Increase of lattice parameters for nanoparticles of some oxide compounds is reported as the particle size goes down. ${ }^{25}$ One reason could be increase of the ratio of surface to volume with decreasing particle size. This results in an increased number of broken $\mathrm{Mn}^{3+}-\mathrm{O}-\mathrm{Mn}^{4+}$ bond causing an increase in the ionic character. Such a situation can give rise to an unpaired electronic orbital at the surface which might repel each other and give rise to increased lattice constant compared to the bulk in order to attain stability. Since the surface contribution increases with decreasing size, the overall lattice expansion also increases. The observance of sudden rise of lattice parameter below $15 \mathrm{~nm}$ is therefore likely a result of the increase of the surface lattice parameters. In addition, due to increased anharmonic vibrations the thermal expansion coefficient of the grain boundary is larger than the bulk. ${ }^{26}$ Therefore the increase of unit cell volume at the surface will be more compared to the bulk. Since the surface to volume ratio increases as the size decreases, the surface lattice parameter contribution to the overall lattice parameter also increases. This explains the overall increase of unit cell volume as the particle size decreases for NP of LSMO. 


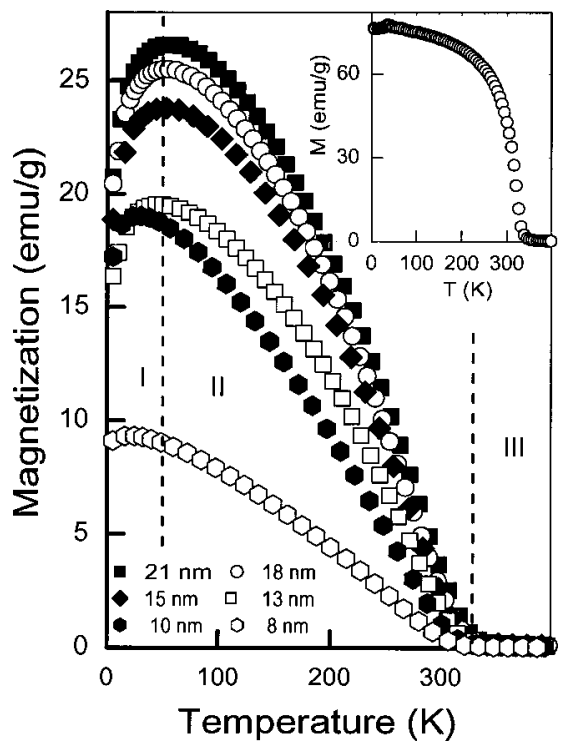

FIG. 3. (a) Zero field magnetization vs temperature curve of $\mathrm{La}_{0.8} \mathrm{Sr}_{0.2} \mathrm{MnO}_{3-\delta}$ at an applied field of $1 \mathrm{kG}$. Region I is the blocked region, II is the superparamagnetic region, and III is the region where the spins of the individual particle also gets disordered. Inset shows the MvT data of a bulk LSMO sample.

\section{B. Magnetic properties}

The zero field cooled temperature variation of the magnetization of the nanoparticles of LSMO is shown in Fig. 3. The inset shows the thermomagnetic data for $53 \mathrm{~nm}$ LSMO which is a ferromagnet with $T_{\mathrm{C}}=325 \mathrm{~K}$. For the other nanoparticles, there is a huge reduction of the magnetization. Such a drastic decrease of the magnetization could be explained by considering a nonmagnetic layer on the surface, the thickness of which increases as the particle size goes down (as is shown later). It is interesting to note that the temperature at which the nanoparticles become paramagnetic is the same for all the particles. This indicates that the magnetization contribution comes from the core and that the stoichiometry of the core is same as that of the $53 \mathrm{~nm}$ LSMO. The magnetization versus temperature plot shows superparamagnetic behavior for all the LSMO nanoparticles. ${ }^{17}$ The nanoparticles of LSMO pass through different magnetic states (shown by dashed line) as the temperature varies from 5 to $400 \mathrm{~K}$. The individual particles are ferromagnetic and the moment of all the randomly oriented particles average out to be zero. At the lowest temperature of $5 \mathrm{~K}$, when the field is applied, the particles try to align in the direction of the field. But since in general, the easy axis and the applied field direction is not same, only a component of the magnetization vector is observed along the field direction. As the temperature is increased, the particles gradually orient along the field increasing the overall magnetization. A peak in therefore observed in the $M(T)$ curve and the temperature at which the peak occurs is called the blocking temperature $T_{\mathrm{B}}$. The region I is therefore the blocking region. In region II $\left(T>T_{\mathrm{B}}\right)$ the temperature is high enough so that the particles no longer are aligned towards the field and start getting disoriented resulting in net decrease of magnetization as temperature increases. But the moment within each individual

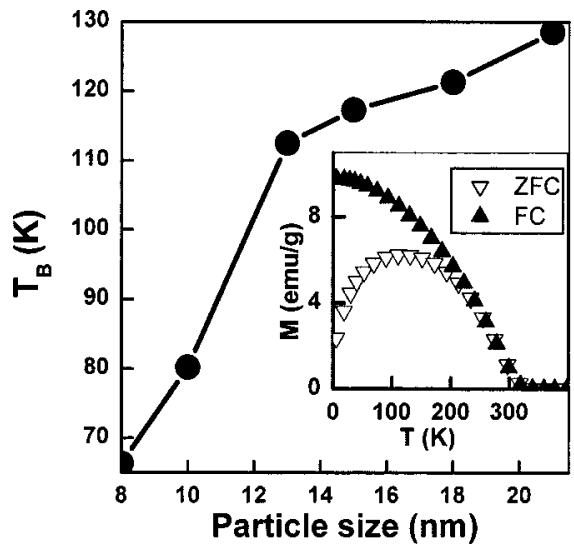

FIG. 4. (a) Variation of blocking temperature as function of particle size. Inset shows a typical $\mathrm{ZFC} / \mathrm{FC}$ curve of $12 \mathrm{~nm}$ particles of $\mathrm{La}_{0.8} \mathrm{Sr}_{0.2} \mathrm{MnO}_{3-\delta}$.

particle is still aligned. This is the superparamagnetic region. Above $T_{\mathrm{C}}$ (region III) the moments in individual particles get disordered and the material becomes paramagnetic.

Figure 4(a) shows a nonmonotonic decrease of the blocking temperature $T_{\mathrm{B}}$ from $130 \mathrm{~K}$ for $23 \mathrm{~nm} \mathrm{NP}$ to $65 \mathrm{~K}$ for the $8 \mathrm{~nm}$ LSMO nanoparticles. The inset of Fig. 4(a) shows a typical $\mathrm{ZFC} / \mathrm{FC}$ magnetization versus temperature curve of the NP. The ZFC/FC curve clearly shows the presence of a peak in ZFC curve, and FC magnetization has an irreversible behavior below the peak temperature. This indicates that below the peak temperature the magnetic system goes onto a disordered blocked state. The blocking temperature had been determined from the peak of the ZFC curve. We also note that the obtained $T_{\mathrm{B}}$ is the average of the $T_{\mathrm{B}}$ 's within the sample because of finite size distribution of the particle size. For an ensemble of noninteracting fine magnetic particles, the blocking temperature is given by

$$
T_{\mathrm{B}}=K_{\mathrm{u}} V / k_{B} \ln \left(\tau_{\mathrm{obs}} / \tau_{\mathrm{o}}\right),
$$

where $K_{\mathrm{u}}$ is the uniaxial anisotropy constant, $V$ is the volume of the particle, and $\tau_{\mathrm{o}}$ is a characteristic time. ${ }^{27}$ It is easily seen from the above equation that $T_{\mathrm{B}}$ increases with particle size. The nonlinear behavior of $T_{\mathrm{B}}$ with the volume [Fig. 4(a)] could be due to additional contributions like dipolar interactions that would cause $T_{\mathrm{B}}$ to vary as $1 / r^{3}$, where $r$ is the distance between two magnetic NP. ${ }^{28}$ The variation of anisotropy constant due to several factors like shape, stress, etc., also contributes to the overall behavior of $T_{\mathrm{B}}$.

The coercivity of the NP at $10 \mathrm{~K}$ is shown in Fig. 5. The coercivity increases from $11 \mathrm{G}$ in $53 \mathrm{~nm}$ LSMO to $443 \mathrm{G}$ for $24 \mathrm{~nm}$ particles of LSMO. But once in the single domain region, the coercivity decreases with decreasing particle size. In $53 \mathrm{~nm}$ LSMO, there are multidomains that make the anisotropy energy small. When particle size is in single domain region, there is a relative increase of the surface compared to the particles that have multidomains. Due to large number of broken bonds and surface stress the surface anisotropy term could become large. Furthermore, in oxide nanoparticles there is a strong interaction between the core and surface. These cause an overall increase of anisotropy constant resulting in increase of coercivity. In the inset the variation of the 


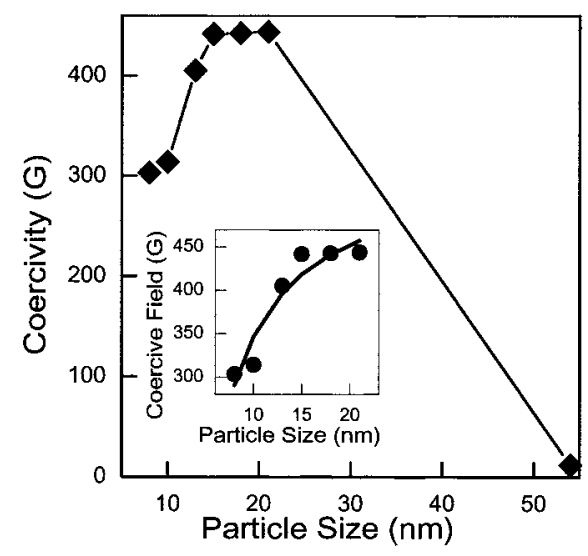

FIG. 5. Particle size dependence of coercivity of $\mathrm{La}_{0.8} \mathrm{Sr}_{0.2} \mathrm{MnO}_{3-\delta}$. Inset shows the change of coercivity as the particle size changes. The dashed curve is fit to the data.

coercive field with the size of single domain LSMO NP is shown. The coercive field grows rapidly from $8 \mathrm{~nm}$ particles to $13 \mathrm{~nm}$ and the rate of increase becomes very low for 15 $\mathrm{nm}$ and higher sized NP. When the particle size becomes very small (single domain region), the energy barrier separating the two energetically degenerate magnetic orientations is small. Thus at any temperature the thermal activation is enough to switch the magnetic orientation. Hence the coercivity decreases with decreasing particle size for single domain particles. For random three-dimensional (3D) uniaxial single domain particles, the coercive field is given by,

$$
H_{\mathrm{C}}(d, T)=H_{\mathrm{C}}(d, 0)\left[1-\left(d_{\mathrm{S}} / d\right)^{3 / 2}\right],
$$

where $d$ and $T$ are the particle size and temperature. ${ }^{29}$ For particles with size greater than $d_{\mathrm{S}}$, the coercive field varies as $d^{-3 / 2}$. Following a similar argument we have done a fit to our data and the result is shown in the inset of Fig. 5. We obtained a fitting equation that is given by

$$
H_{\mathrm{C}}(d, T)=542.77\left[1-4.824(1 / d)^{1.125}\right] .
$$

Clearly instead of $d^{-1.5}$ variation we observe a $d^{-1.125}$ behavior. The maximum coercive field obtained is $543 \mathrm{G}$ which is realized in the limiting case of $d \rightarrow \infty$. The critical size is obtained as $d_{\mathrm{S}}=6 \mathrm{~nm}$. For all small particles of $\mathrm{La}_{0.8} \mathrm{Sr}_{0.2} \mathrm{MnO}_{3-\delta}$ with $d$ greater than $6 \mathrm{~nm}, H_{\mathrm{C}}>H_{\mathrm{C}}(6 \mathrm{~nm}$, $T)$. It may, however, be noted that there exists an upper limit for the size of the particles above which multidomain starts forming and causes the coercive field to decrease. As a result coercive field of $543 \mathrm{G}$ is never realized.

Figure 6 shows the variation of the saturation magnetization $M_{\mathrm{S}}$ as function of NP size at a temperature of $5 \mathrm{~K}$. The decrease of $M_{\mathrm{S}}$ with decreasing NP size could be explained by considering the presence of a noncollinear magnetic outermost shell of thickness " $t$ " that increases relatively with decreasing particle size. ${ }^{6,15,16}$ As the particle size decreases the ratio of thickness of the outer layer to the particle size increases. Considering that the outer layer has no net moment, the net moment decreases as the particle size goes down. In the inset of Fig. 6 we have plotted the varia-

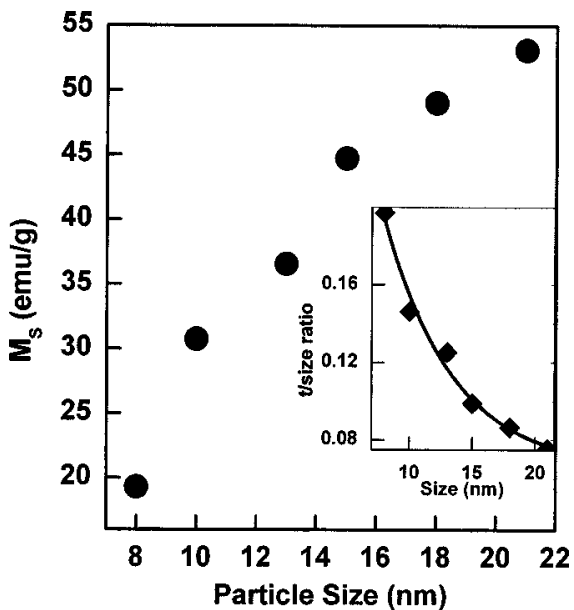

FIG. 6. Effect of particle size on the saturation magnetization. The inset shows the ratio of the outer shell thickness to particle size vs the particle size. The dashed curve is fit to the data.

tion of the $t$ /size ratio with the particle size. An exponential increase of the $t$ /size ratio is observed in accordance with the fitting equation

$$
t / \text { size }=\text { Const }+0.55046^{*} \exp (- \text { size } / 5.51518),
$$

where Const $=0.0648$ is the baseline offset.

It might be noted that a direct consequence of the increase of the ratio is increase of resistivity. Due to increased ratio of the noncollinear spins, the resistivity due to spin scattering also increases resulting in an increased resistivity. Increase of resistivity by decreasing particle size has been reported by many authors. ${ }^{14,15,16}$

In order to understand the behavior of NP in the presence of field, applied magnetic field dependent experiments were performed for LSMO with particle size of $12 \mathrm{~nm}$. Figure 7 shows the variation of blocking temperature $T_{\mathrm{B}}$ versus applied magnetic field. The blocking temperature decreases with increasing applied magnetic field. Since the blocking temperature equals the energy that the moment requires to get aligned along the field, so in addition when an external field is applied, the energy of the barrier decreases to $K V$ $-\mu H$, where, $K$ is anisotropy constant. The moment there-

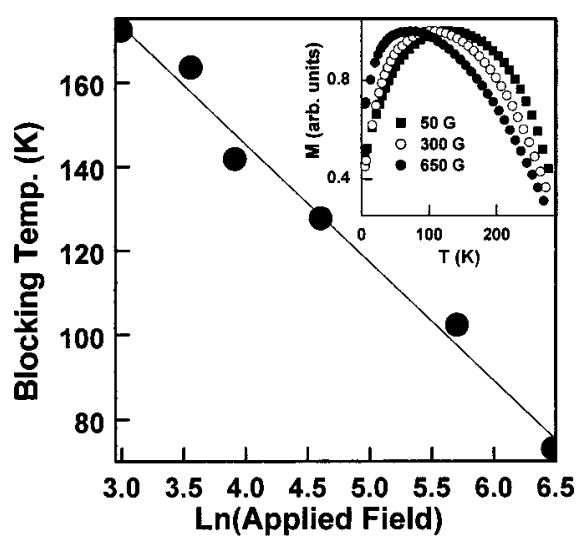

FIG. 7. The dependence of Blocking temperature on the applied magnetic field for $12 \mathrm{~nm}$ LSMO particles. Inset shows the change in the magnetization curve shape as function of the applied magnetic field. 


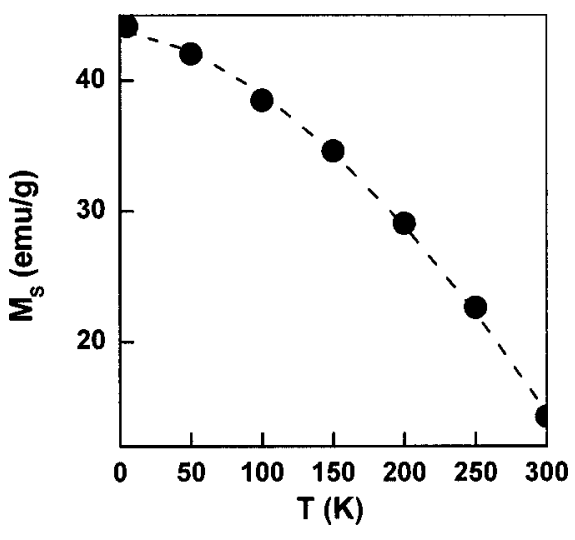

FIG. 8. Saturation magnetization as a function of temperature for $12 \mathrm{~nm}$ LSMO particles. The dashed curve is a power law fit of the form $M_{\mathrm{S}}$ $=M_{0}\left(1-B T^{\alpha}\right)$ to the data points.

fore requires less energy to get itself aligned along the field. As a consequence the blocking temperature also decreases. A logarithmic behavior of $T_{\mathrm{B}}$ with applied magnetic field is reported by Li et al. ${ }^{17}$ The $T_{\mathrm{B}}$ in our case have also been fitted well by

$$
T_{\mathrm{B}}=257.4-28.1 \ln (\text { Applied Field }) \text {. }
$$

However, in case of $\mathrm{Co}-\mathrm{Ag}$ system it has been found that $T_{\mathrm{B}}$ has a quadratic dependence on $H \cdot{ }^{30}$ At this stage we are not sure as to why the difference has come up. From the above fitted equation it could be seen that at very low field the blocking temperature of LSMO nanoparticles are very high $(\approx 250 \mathrm{~K})$. This therefore could have important technological application. In the inset we have shown how the shape of the curve changes as the applied field is increased. Apart from the shift of the peak towards lower temperatures at higher fields, the curves broaden out at lower applied fields. The broadening of the curves at low fields reflects the distribution of the particle sizes. At low enough fields, the energy gained due to the field is small and the unblocking takes place mainly due to temperature increase. Consequently the rate of unblocking is slow. Eventually the temperature is high enough so that all the particles are unblocked and the superparamagnetism is observed. The rate is increased as the contribution of field to the unblocking process increases.

Figure 8 shows the behavior of the saturation magnetization $M_{\mathrm{S}}$ versus temperature for $12 \mathrm{~nm}$ LSMO nanoparticles. The $M_{\mathrm{S}}$ value is obtained by extrapolating the applied magnetic field $H \rightarrow \infty$ and finding the magnetization from the $M(H)$ curve. In the case of Heisenberg spin clusters, the temperature dependence of magnetization including finite size effects is given by a power law of the form ${ }^{20}$

$$
M_{\mathrm{S}}=M_{0}\left(1-B T^{\alpha}\right) \text {. }
$$

A fit to the magnetization data is shown in Fig. 8 (dashed curve) with $M_{0}=43.76 \mathrm{emu} / \mathrm{g}, B=5.1 \times 10^{-5} \mathrm{~K}^{-1.66}$, and $\alpha=1.66$. The magnetization therefore does not follow Bloch's $T^{3 / 2}$ law but rather it follows $T^{1.66}$ law. The slightly greater value of $\alpha$ compared to the bulk value (3/2) is consistent with the theory as described in Ref. 31 and is due to effect of small particle size. Therefore, $\alpha$ increases as the particle size decreases. The value of $B$ is greater than the bulk value. This is most likely due to an increased interaction among the neighboring spins resulting in an increased moment in the nanoparticles as compared to the bulk sample.

\section{CONCLUSIONS}

The structural and magnetic properties of the nanoparticles of $\mathrm{La}_{0.8} \mathrm{Sr}_{0.2} \mathrm{MnO}_{3-\delta}$ have been studied in great detail. The properties of nano-LSMO have been found to be influenced increasingly by the surface properties as the particle size goes down. The particles show increasing strain hardening as the size of the particles decrease. As a result there exists a minimum particle size below which smaller nanoparticles are not possible to obtain using ball mill method. The minimum particle size depends on various parameters of the ball mill. The unit cell volume increases as the particle size decreases. The evolution of microstrain as a function of particle size shows a peak at around particle size of $15 \mathrm{~nm}$ and is due to change in the contribution to the strain from dislocation densities to gliding action along grain boundaries. Thermo-magnetic measurements show that fine particles of LSMO exhibit superparamagnetic behavior with a blocking temperature that has a nonlinear behavior as function of the particle size. A sharp decrease in magnetization for nanogranular LSMO indicates formation of magnetically dead layer at the surface. The thickness of such a magnetically dead layer had been found to have a logarithmic dependence on the particle size. The coercivity of the nanoparticles of LSMO increases manifold as the LSMO particle size decrease from $53 \mathrm{~nm}$ to $21 \mathrm{~nm}$. This indicates that nano-LSMO could be very important from technological point of view. The temperature dependence of magnetization in the case of nano-LSMO follows a power law of the form $T^{\alpha}$ where $\alpha>3 / 2$. Thus collective excitations are observed but due to finite size effects they do not follow Bloch law.

From our study it becomes evident that in the study of nanoparticles of LSMO the surface plays a very important role. Since at the surface there are many broken symmetries, so the properties are very different. It is therefore very important to characterize the surface of the nanoparticles of LSMO. Further experiment is needed to understand the spin disorder mechanism at the surface. It would be interesting to do x-ray scattering studies on the nanoparticles to gain insight into the surface.

${ }^{1}$ Nanomagnetism, edited by A. Hernando (Kluwer Academic, Dordrecht, MA, 1993).

${ }^{2}$ Nanophase Materials: Synthesis, Properties, Applications, edited by G. C. Hadjipanayis and R. W. Siegel (Kluwer, Dordrecht, 1994).

${ }^{3}$ C. L. Chein, J. Appl. Phys. 69, 5267 (1991).

${ }^{4}$ Paolo Allia, Marco Coisson, Paola Tiberto, Franco Vinai, Marcelo Knobel, M. A. Vovak, and W. C. Nunes, Phys. Rev. B 64, 144420 (2001).

${ }^{5}$ J. C. Denardin, A. L. Brandl, M. Knobel, P. Pannisod, A. B. Pakhomov, H. Liu, and X. X. Zhang, Phys. Rev. B 65, 064422 (2002).

${ }^{6}$ S. Gangopadhyay, G. C. Hadjipanayis, B. Dale, C. M. Sorensen, K. J. Klabunde, V. Papaefthymiou, and A. Kostikas, Phys. Rev. B 45, 9778 (1992).

${ }^{7}$ R. H. Kodama, A. E. Berkowitz, E. J. McNiff, and S. Foner, Phys. Rev. Lett. 77, 394 (1996).

${ }^{8}$ Sanshiro Sako, Yoshifumi Umemura, Kazunari Ohshima, Masahiro Sakai, and Shunji Bandow, J. Phys. Soc. Jpn. 65, 280 (1995).

${ }^{9}$ Colossal Magnetoresistive Oxides, edited by Y. Tokura (Gordon and Breach, London, 1999). 
${ }^{10}$ C. Zener, Phys. Rev. 82, 403 (1951).

${ }^{11}$ A. J. Millis, P. B. Littlewood, and B. Shraiman, Phys. Rev. Lett. 74, 5144 (1995).

${ }^{12}$ H. Y. Hwang, S.-W Cheong, N. P. Ong, and B. Battlogg, Phys. Rev. Lett. 77, 2041 (1996).

${ }^{13}$ A. Gupta, G. Q. Gong, G. Xiao, P. R. Duncombe, P. Trouilloud, P. Lecoeur, Y. Y. Wang, V. P. Dravid, and J. Z. Sun, Phys. Rev. B 54, R15629 (1996).

${ }^{14}$ J. M. D. Coey, J. Appl. Phys. 85, 5576 (1999).

${ }^{15}$ L. L. Balcells, J. Fontcuberta, B. Martinez, and X. Obradors, Phys. Rev. B 58, R14697 (1998).

${ }^{16}$ M. A. Lopez-Quintela, L. E. Hueso, J. Rivas, and F. Rivadulla, Nanotechnology 14, 212 (2003).

${ }^{17}$ Run-Wei Li, Han Xiong, Ji-Rong Sun, Qing-An Li, Zhi-Hong Wong, Jian Zhang, and Bao-Gen Shen, J. Phys.: Condens. Matter 13, 141 (2000).

${ }^{18}$ H. M. Rietveld, J. Appl. Crystallogr. 2, 65 (1969).

${ }^{19}$ G. K. Williamson and W. H. Hall, Acta Metall. 1, 22 (1953).

${ }^{20}$ Davor Balzar and Hassel Ledbetter, J. Appl. Crystallogr. 26, 97 (1993); Davor Balzar, ibid. 25, 559 (1992) (http://www.boulder.nist.gov/div853/ balzar/breadth.htm).
${ }^{21}$ M. A. Myers and K. K. Chawla, Mechanical Metallurgy (Prentice Hall, Englewood Cliffs, NJ, 1984).

${ }^{22}$ E. Hellstern, H. J. Fecht, Z. Fu, and W. L. Johnson, J. Appl. Phys. 65, 305 (1989).

${ }^{23}$ Y. H. Zhao, K. Lu, and K. Zhang, Phys. Rev. B 66, 085404 (2002).

${ }^{24}$ J. Karch, R. Berringer, and H. Gleiter, Nature (London) 330, 556 (1987).

${ }^{25}$ Pushan Ayyub, V. R. Palkar, Soma Chattopadhyay, and Manu Multani, Phys. Rev. B 51, 6135 (1995).

${ }^{26}$ H. J. Klam, H. Hahn, and H. Gleiter, Acta Metall. 35, 2101 (1987).

${ }^{27}$ L. Néel, Ann. Geophys. 5, 99 (1949).

${ }^{28}$ Weili Luo, Sidney R. Nagel, T. F. Rosenbaum, and R. E. Rosensweig, Phys. Rev. Lett. 67, 2721 (1991).

${ }^{29}$ E. F. Kneller and F. E. Luborsky, J. Appl. Phys. 34, 656 (1963).

${ }^{30}$ Y. D. Zhang, J. I. Budnick, W. A. Hines, C. L. Chien, and J. Q. Xiao, Appl. Phys. Lett. 72, 2053 (1998).

${ }^{31}$ P. V. Hendriksen, S. Linderoth, and P.-A. Lindgard, Phys. Rev. B 48, 7259 (1993). 